\title{
Simoctocog Alfa (Nuwiq) in Previously Untreated Patients with Severe Haemophilia A: Final Results of the NuProtect Study
}

\author{
Ri J. Liesner ${ }^{1}$ Aby Abraham ${ }^{2}$ Carmen Altisent ${ }^{3}$ Mark J. Belletrutti ${ }^{4}$ Manuel Carcao $^{5}$ \\ Manuela Carvalho ${ }^{6}$ Hervé Chambost ${ }^{7}$ Anthony K. C. Chan ${ }^{8}$ Leonid Dubey ${ }^{9}$ Jonathan Ducore ${ }^{10}$ \\ Michael Gattens ${ }^{11}$ Paolo Gresele ${ }^{12}$ Yves Gruel ${ }^{13}$ Benoit Guillet ${ }^{14}$ Victor Jimenez-Yuste ${ }^{15}$ \\ Lidija Kitanovski $^{16}$ Anna Klukowska ${ }^{17}$ Sunil Lohade $^{18}$ Maria Elisa Mancuso $^{19}$ Johannes Oldenburg ${ }^{20}$ \\ Anna Pavlova ${ }^{20}$ Berardino Pollio ${ }^{21}$ Marianne Sigaud ${ }^{22}$ Vladimir Vdovin ${ }^{23}$ Kateryna Vilchevska ${ }^{24}$ \\ John K. M. Wu ${ }^{25}$ Martina Jansen ${ }^{26}$ Larisa Belyanskaya ${ }^{27}$ Olaf Walter ${ }^{27}$ Sigurd Knaub ${ }^{27}$
}

Ellis J. Neufeld ${ }^{28}$

${ }^{1}$ Great Ormond Street Hospital for Children NHS Trust Haemophilia Centre, NIHR GOSH BRC, London, United Kingdom

2 Department of Hematology, Christian Medical College, Vellore, India

${ }^{3}$ Unitat d'Hemofilia, Hospital Vall D'Hebron, Barcelona, Spain

4 Pediatric Hematology, Department of Pediatrics, University of Alberta, Edmonton, Canada

${ }^{5}$ Division of Haematology/Oncology and Child Health Evaluative Sciences, Department of Paediatrics, Research Institute, Hospital for Sick Children, Toronto, Canada

${ }^{6}$ Congenital Coagulopathies Reference Centre, São João University Hospital Centre, Porto, Portugal

${ }^{7}$ AP-HM, Department of Pediatric Hematology Oncology, Children Hospital La Timone, Aix Marseille Univ, INSERM, INRA, C2VN, Marseille, France

8 Division of Pediatric Hematology/Oncology, McMaster University, Hamilton, Canada

${ }^{9}$ Department of Pediatrics, Western Ukrainian Specialized Children's Medical Centre, Lviv, Ukraine

${ }^{10}$ Department of Pediatrics, University of California Davis Medical Center, Sacramento, United States

${ }^{11}$ Department of Paediatric Haematology and Oncology, Addenbrooke's Hospital, Cambridge University Hospital NHS Foundation Trust, Cambridge, United Kingdom

12 Department of Medicine and Surgery, University of Perugia, Perugia, Italy

${ }^{13}$ Centre Régional de Traitement de l'Hémophilie, Hôpital Trousseau, Tours, France

${ }^{14}$ Haemophilia Treatment Centre, Univ Rennes, CHU Rennes, Inserm, EHESP, Irset (Institut de recherche en santé, environnement et travail) - UMR_S 1085, Rennes, France
Address for correspondence Ri J. Liesner, MA, MBBChir, MD, FRCP, FRCPath, MRCPCH, Great Ormond Street Hospital for Children NHS Trust Haemophilia Centre, NIHR GOSH BRC, London, United Kingdom (e-mail: Ri.Liesner@gosh.nhs.uk).

${ }^{15}$ Servicio de Hematología, Hospital Univeristario La Paz, Autónoma University, Madrid, Spain

16 Department of Haemato-Oncology, University Medical Center Ljubljana, Ljubljana, Slovenia

${ }^{17}$ Department of Pediatrics, Haematology and Oncology, Warsaw Medical University, Warsaw, Poland

${ }^{18}$ Department of Hematology, Sahyadri Speciality Hospital, Pune, India

${ }^{19}$ Center for Thrombosis and Hemorrhagic Diseases, Humanitas Clinical and Research Center - IRCCS, Rozzano, Milan, Italy

${ }^{20}$ Institute of Experimental Haematology and Transfusion Medicine, University Clinic Bonn, Bonn, Germany

${ }^{21}$ Department of Transfusion Medicine, Regina Margherita Children Hospital of Turin, Turin, Italy

${ }^{22}$ Centre Régional de Traitement de l'Hémophilie, University Hospital of Nantes, Nantes, France

${ }^{23}$ Department of Hematology, Morozovskaya Children's Hospital, Moscow, Russian Federation

${ }^{24}$ Department of Hematology, State Institution "Institute of Urgent and Reconstructive Surgery named after V.K. Gusak of National Academy of Medical Sciences of Ukraine," Donetsk, Ukraine

25 British Columbia Children's Hospital, Vancouver, Canada

26 Octapharma Pharmazeutika Produktionsges.mbH, Vienna, Austria

27 Octapharma AG, Lachen, Switzerland

${ }^{28}$ St. Jude Children's Research Hospital, Memphis, Tennessee, United States

Thromb Haemost 2021;121:1400-1408.

received

October 14, 2020

accepted after revision

December 4, 2020

published online

February 13, 2021
DOI https://doi.org/

$10.1055 / \mathrm{s}-0040-1722623$.

ISSN 0340-6245.

\section{(c) 2021. The Author(s).}

This is an open access article published by Thieme under the terms of the Creative Commons Attribution License, permitting unrestricted use, distribution, and reproduction so long as the original work is properly cited. (https://creativecommons.org/licenses/by/4.0/)

Georg Thieme Verlag KG, Rüdigerstraße 14, 70469 Stuttgart, Germany 
Abstract

\section{Keywords}

- coagulation

- FVIII inhibitors

- haemophilia
Introduction FVIII inhibitor development is the most serious contemporary treatment complication in haemophilia A, particularly in previously untreated patients (PUPs). No inhibitors developed in clinical trials in previously treated patients treated with simoctocog alfa (Nuwiq), a fourth-generation recombinant FVIII produced in a human cell line.

Methods The NuProtect study investigated the immunogenicity of simoctocog alfa in PUPs. NuProtect was a prospective, multinational, open-label, non-controlled, phase III study. PUPs with severe haemophilia A (FVIII:C $<1 \%$ ) of any age and ethnicity were treated with simoctocog alfa for 100 exposure days or a maximum of 5 years. Patients were true PUPs without prior exposure to FVIII concentrates or blood components. Inhibitor titres were measured with the Nijmegen-modified Bethesda assay; cut-off for positivity was $0.6 \mathrm{BU} \mathrm{mL} \mathrm{m}^{-1}$ ( $\geq 0.6$ to $<5$ low-titre, $\geq 5$ high titre).

Results A total of 108 PUPs with a median age at first treatment of 12.0 months (interquartile range: 8.0-23.5) were treated with simoctocog alfa. F8 mutation type was known for 102 patients (94.4\%) of whom 90 (88.2\%) had null F8 mutations and 12 (11.8\%) had non-null mutations. Of 105 PUPs evaluable for inhibitor development, 28 (26.7\%) developed inhibitors; 17 high titre (16.2\%) and 11 low titre (10.5\%). No PUPs with non-null F8 mutations developed inhibitors.

Conclusion In the NuProtect study, the rate of inhibitor development in PUPs with severe haemophilia A treated with simoctocog alfa was lower than the rate reported for hamster-cell-derived recombinant factor VIII products in other recent clinical trials. No inhibitors were reported in PUPs with non-null F8 mutations.

\section{Introduction}

Haemophilia $\mathrm{A}$ is an X-linked bleeding disorder that is usually diagnosed in infancy, especially in severe cases (factor VIII coagulant activity [FVIII:C] $<1 \%$ of normal). ${ }^{1}$ Patients with haemophilia A require life-long treatment to prevent or control bleeding. ${ }^{2,3}$

When FVIII treatment is started, previously untreated patients (PUPs) with severe haemophilia A face a period of high risk for development of FVIII-neutralising antibodies (inhibitors), which interfere with the haemostatic function of FVIII treatment. The highest risk is present usually during the first 20 exposure days (EDs) to exogenous FVIII but a residual risk persists until the completion of $75 \mathrm{EDs}^{4,5}$ Inhibitors typically develop in up to $40 \%$ of $\mathrm{PUPs}^{6}$ and are widely considered the most serious treatment-related complication of haemophilia A due to the detrimental impact on bleeding rates, mortality, quality of life and treatment costs. ${ }^{7-10}$ Remaining free from inhibitors is important to enable effective FVIII treatment for bleeding events or surgery, as well as to remain eligible for potential future treatment options such as gene therapy. ${ }^{6,11}$

Several patient- and treatment-related risk factors for inhibitor development have been identified, such as severity of haemophilia A, a family history of inhibitors, ethnicity, type of F8 mutation, polymorphisms of immune response genes, treatment intensity and product type. ${ }^{4,12-14}$ Whereas some reports suggest an increased inhibitor risk associated with the use of recombinant (r) FVIII compared with plasma- derived (pd) FVIII concentrates, ${ }^{15-17}$ other reports suggest no difference in inhibitor risk with respect to product type. ${ }^{18-20}$ The SIPPET study was the only prospective, randomised, controlled study to compare the immunogenicity of pdFVIII versus rFVIII (all derived from hamster cell lines). ${ }^{21}$ The cumulative incidence of all and high-titre inhibitors in patients treated with pdFVIII was $26.8 \%$ (95\% confidence interval $[\mathrm{CI}]$ : $18.4-35.2 \%$ ) and $18.6 \%$ (95\% CI: $11.2-26.0 \%$ ), respectively. In patients treated with $\mathrm{FFVIII}$, the cumulative incidence of all and high-titre inhibitors was 44.5\% (95\% CI: $34.7-54.3 \%$ ) and $28.4 \%$ (95\% CI: 19.6-37.2\%), respectively. ${ }^{21}$ As SIPPET enrolled patients between 2010 and 2014, rFVIII products licensed after 2014 were not included in the study.

Simoctocog alfa (Nuwiq; Octapharma AG) is a fourthgeneration rFVIII product ${ }^{22}$ manufactured using a human cell line without chemical modification or protein fusion, with the aim of reducing inhibitor development by replicating the native human FVIII protein and avoiding incorporation of potentially immunogenic elements of animal cell origin. ${ }^{23-26}$ It contains only human post-translational modifications ${ }^{24}$ and is free of any added human or animal impurities. ${ }^{23,26}$ Simoctocog alfa is fully sulphated at Tyr 1680 , which confers a high binding affinity for its natural carrier protein, von Willebrand factor (VWF) ${ }^{24,25}$ These properties of simoctocog alfa may lower its immunogenic potential by shielding potentially antigenic epitopes from recognition by the immune system and inhibiting uptake of FVIII by antigen-presenting cells. ${ }^{12}$ Additionally, simoctocog alfa contains only human glycans and is devoid of potentially immunogenic glycan epitopes present in rFVIII 
products derived from hamster cell lines. ${ }^{27,28} \mathrm{~A}$ combination of high VWF affinity and absence of antigenic glycans has the potential to reduce the overall immunogenic challenge. The efficacy and safety of simoctocog alfa were demonstrated in studies of previously treated patients (PTPs) with severe haemophilia A, and no inhibitors were reported in 201 PTPs, including 59 PTPs below 12 years of age. ${ }^{29}$

The NuProtect study was initiated in 2013 to assess the immunogenicity of simoctocog alfa in patients without any previous exposure to FVIII concentrates or any blood products containing FVIII (true PUPs) who were treated with simoctocog alfa for $100 \mathrm{EDs}$ or up to 5 years, whichever came first. Here we report the final inhibitor data of the NuProtect study; efficacy and safety data will be reported separately.

\section{Methods}

Details of the study design and clinical assessments have been published previously ${ }^{30}$ and are provided in brief here with respect to inhibitor development.

\section{Study Design and Patients}

The NuProtect study (www.clinicaltrials.gov NCT01712438; EudraCT 2012-002554-23) was a prospective, multicentre, multinational, open-label, non-controlled, phase III study. Male PUPs with severe haemophilia A (FVIII:C $<1 \%$ ) of any age and ethnicity were enrolled and treated with simoctocog alfa from the first ED. Exclusion criteria included previous exposure to any FVIII concentrates or blood components, diagnosis of any coagulation disorder other than haemophilia A, concomitant treatment with systemic immunosuppressive drugs, participation in other interventional clinical studies currently or within previous 4 weeks and severe liver or kidney disease. The trial was approved by all relevant independent ethics committees and institutional review boards and was conducted in accordance with the ethical principles of the Declaration of Helsinki. Written informed consent was provided by the parent/legal guardian of all participants.

\section{Study Treatment}

Patients received simoctocog alfa for prophylaxis or on-demand treatment, as well as for the treatment of breakthrough bleeding episodes during prophylaxis and to cover surgical procedures. The type of treatment and the dose were determined by investigators based on the clinical situation of the patient.

The recommended dose for prophylaxis was 20 to $50 \mathrm{IU}$ FVIII $\mathrm{kg}^{-1}$ and patients could switch between on-demand and prophylactic treatment during the study. Use of FVIII concentrates other than simoctocog alfa was prohibited, except in emergency situations. Patients permanently switching to another FVIII product within the study participation period were assessed as treatment failures in the efficacy analyses. The treatment period was 100 EDs or 5 years, whichever occurred first.

\section{Inhibitor Analysis}

The primary endpoint of the study was the incidence of antiFVIII inhibitors after simoctocog alfa administration. Inhibitor titres were measured in plasma by the Nijmegen-modified Bethesda assay (cut-off: 0.6 Bethesda units $[\mathrm{BU}] \mathrm{mL}^{-1}$ ). ${ }^{31}$

Inhibitor activity was determined at a central laboratory (LabCorp Clinical Trials, Colorado, United States) at baseline (screening), every 3 to 4 EDs until ED 20, every 10 to 12 EDs or every 3 months \pm 2 weeks after ED 20 and at study completion. Additionally, inhibitor activity was tested at any time during the study if inhibitor development was suspected. In the case of a positive inhibitor result, a retest of the first sample was done and a second blood sample was drawn and tested at the central laboratory. Inhibitors were confirmed if the retest or second sample was positive or a subsequent test at any time in the study was positive. The day of the first positive inhibitor test was used to calculate the time to inhibitor development. The definitions for inhibitor thresholds were $\geq 0.6$ to $<5 \mathrm{BU} \mathrm{mL}^{-1}$ for low-titre and $\geq 5 \mathrm{BU} \mathrm{mL}^{-1}$ for high-titre inhibitors. Inhibitors were regarded as transient if they occurred without any clinical symptoms, required no increase in FVIII dosing and decreased to $<0.6 \mathrm{BU}$ $\mathrm{mL}^{-1}$ within a period of 6 months after first detection. Patients who developed a non-transient, low- or high-titre inhibitor were offered the option to start immune tolerance induction (ITI) treatment with simoctocog alfa within the study; ITI data are not reported here.

The incidence of FVIII inhibitors was examined according to patient-related variables: race, family history of inhibitors and F8 mutation type (performed by J. Oldenburg and A. Pavlova, University Clinic Bonn, Bonn, Germany). Intron 22 inversions, intron 1 inversions, nonsense mutations, splice site mutations, small duplications, small deletions (excluding in-frame and within a poly-A run) and large deletions were classified as null mutations. All other mutations were classified as non-null mutations. The effect of treatment-related variables, including treatment regimen (prophylaxis vs. on-demand), surgery, peak treatment moments ( $\geq 3$ subsequent days with FVIII dosing and/or at least 1 day with prophylactic doses of $>50 \mathrm{IU} \mathrm{kg}^{-1}$ ) and dose ( $<40$ vs. $\geq 40 \mathrm{IU} \mathrm{kg}^{-1}$ day $^{-1}$ ), were also examined.

\section{Statistical Analyses}

No inferential analysis involving formal testing was planned in this non-controlled study. Therefore, no formal sample size estimation was performed, but the sample size was chosen to satisfy European Medicines Agency guidelines current at the time the study was initiated (EMA/CHMP/ BPWP/144533/2009).

Statistical analyses were exploratory and were performed by Clinipace (Marburg, Germany). Absolute and cumulative inhibitor incidences were calculated. Cumulative incidences and 95\% CIs were calculated using Kaplan-Meier methods relative to the population size and accounted for changes over time in the number of patients remaining at risk of developing inhibitors. 


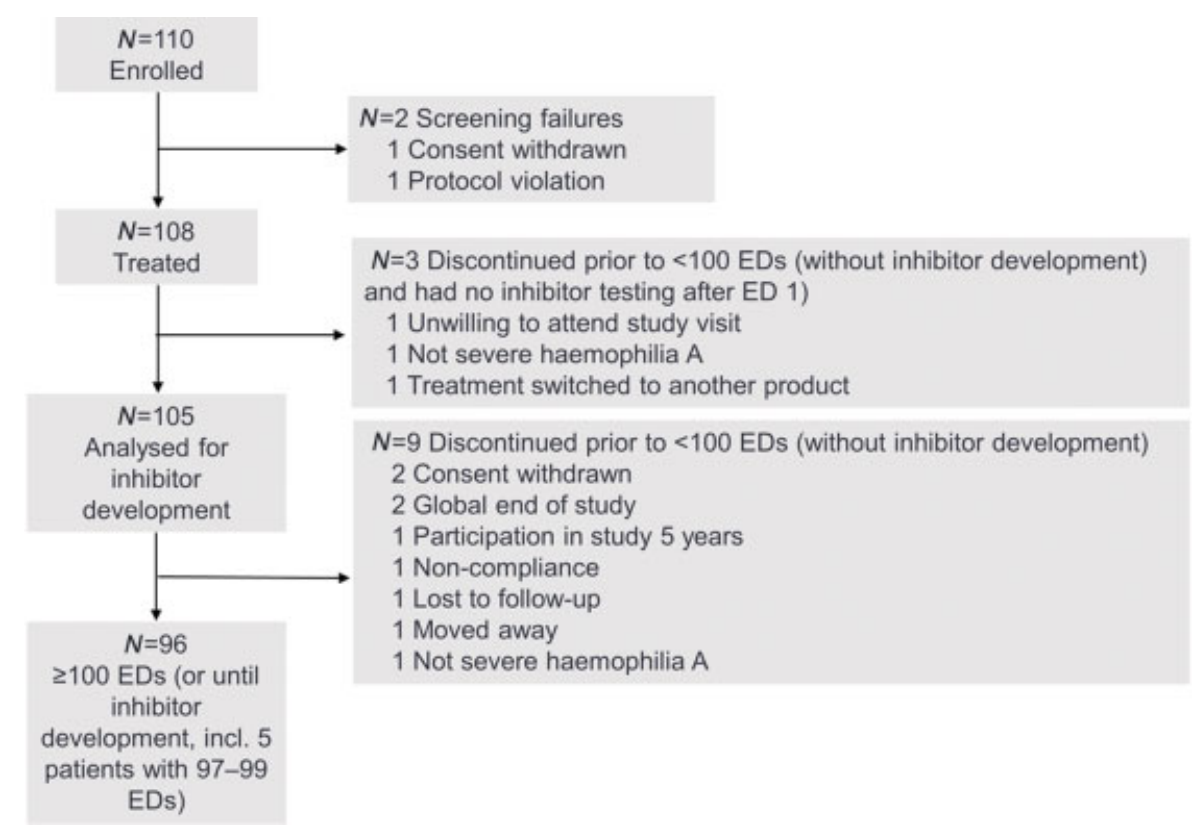

Fig. 1 Patient disposition.

\section{Results}

\section{Patients}

The study was conducted at 38 sites in 17 countries. A total of 110 patients were screened, 108 (98\%) were treated with simoctocog alfa and 105 (97\%) underwent inhibitor testing at least once during the study (-Fig. 1). Three patients discontinued the study after $1 \mathrm{ED}$ without an inhibitor test and were not included in the inhibitor analysis. A total of 105 patients underwent inhibitor testing and were included in the immunogenicity analyses. The 105 patients were treated for a median of 100 EDs (interquartile range [IQR]: 15-102), excluding treatment for ITI. Ninety-six patients completed the study, i.e. received simoctocog alfa for $\geq 100$ EDs without developing an inhibitor (including 5 patients with 97-99 EDs due to miscounting) and/or developed an inhibitor. Five patients who developed a transient inhibitor subsequently reached $\geq 100$ EDs. Nine patients discontinued the study after a median of 14 EDs (IQR: 5-32) without developing an inhibitor.

Baseline demographics are shown in - Table 1. At ED 1, the median age of enrolled patients at first treatment was 12.0 months (IQR: 8.0-23.5), and 82 (75.9\%) were aged $\leq 24$ months. F8 mutation type was known for $102 / 108$ (94.4\%) and 90 of them (88.2\%) had null F8 mutations. Patients with null mutations received their first treatment at a median age of 12.0 months (IQR: 8.0-22.8) and those with non-null mutations at a median of 14.0 months (IQR: 10.5-21.3). A family history of haemophilia was present in 42 (38.9\%) patients of whom $13(31.0 \%)$ also had a family history of inhibitors.

\section{Inhibitor Development}

Of the 105 patients who underwent inhibitor testing, 28 (26.7\%) developed inhibitors, which were high titre in 17 (16.2\%) and low titre in 11 (10.5\%). Five of the 11 patients with low-titre inhibitors had transient inhibitors with titres becoming undetectable without the need for treatment regimen modification and all were subsequently treated for $\geq 100$ EDs. In 97 patients who were treated for $\geq 75$ EDs (or developed an inhibitor), the absolute inhibitor incidences were $28.9,17.5$ and $11.3 \%$, for all, high- and low-titre inhibitors, respectively.

The cumulative inhibitor incidence was $27.9 \%$ (95\% CI: $19.1-36.7 \%)$ for all inhibitors, $17.6 \%$ (95\% CI: 10.0-25.3\%) for high-titre inhibitors and $12.3 \%$ (95\% CI: 5.5-19.2\%) for lowtitre inhibitors (-Fig. 2).

The median (IQR) peak (maximum) inhibitor titre was 154.8 (38.2-300.5) BU $\mathrm{mL}^{-1}$ during the study for high-titre inhibitors and $3.0(2.3-4.0) \mathrm{BU} \mathrm{mL}^{-1}$ for low-titre inhibitors. The median (range) time to inhibitor development was 11.0 (4-34) EDs for all inhibitors (9.0 [4-24] EDs and 12.0 [6-34] EDs for high- and low-titre inhibitors, respectively). Inhibitors developed after ED 20 in only 3 cases ( 1 high titre [ED 24], 2 low titre [ED 25 and ED 34]).

Of 90 patients with null F8 mutations, 27 (30.0\%) developed inhibitors of which 17 (18.9\%) were high titre (no mutation was found for one patient with a low-titre inhibitor); the cumulative incidence was 30.9\% (95\% CI: $21.2-40.6 \%$ ) for all inhibitors and $20.3 \%$ (95\% CI: 11.6-28.9\%) for high-titre inhibitors ( - Fig. 3). No patients with non-null F8 mutations $(n=12)$ developed inhibitors. Patients with large $F 8$ deletions $(n=5)$ had the highest inhibitor incidence (80\%) (-Fig. 4). Patients with a family history of inhibitors $(n=13)$ had a higher incidence of inhibitors (46.2\%) compared with those without $(n=92)$ (23.9\%; - Fig. 4). Patients aged 1 to 6 months (37.5\%; $n=8)$ and aged $>6-12$ months $(36.2 \% ; n=47)$ at first treatment had a higher incidence of inhibitors compared with patients aged $>12-24$ months $(20.0 \% ; n=25)$ or $>24$ months (12.5\%; $n=24$ ) (-Fig. 4 ).

Treatment-related factors (treatment regimen, surgery, peak treatment moments and dose) did not have a significant 
Table 1 Patient demographics

\begin{tabular}{|c|c|}
\hline Parameter & $\begin{array}{l}\text { All patients } \\
(N=108)\end{array}$ \\
\hline $\begin{array}{l}\text { Age at first treatment, } \\
\text { mo, median (IQR) }\end{array}$ & $\begin{array}{l}12.0 \\
(8.0-23.5)\end{array}$ \\
\hline \multicolumn{2}{|l|}{ Age at first treatment, $N(\%)$} \\
\hline$<1 \mathrm{mo}$ & $1(0.9)$ \\
\hline $1-6 \mathrm{mo}$ & $9(8.3)$ \\
\hline$>6-12 \mathrm{mo}$ & $47(43.5)$ \\
\hline$>12-24 \mathrm{mo}$ & $25(23.1)$ \\
\hline$>24 \mathrm{mo}$ & $26(24.1)$ \\
\hline \multicolumn{2}{|l|}{ Race, $N(\%)$} \\
\hline White & $89(82.4)$ \\
\hline Asian & $14(13.0)$ \\
\hline Native American/Alaska native & $1(0.9)$ \\
\hline Other & $4(3.7)$ \\
\hline \multicolumn{2}{|l|}{ Gene mutation defect, $N(\%)$} \\
\hline Intron 1 inversion & $3(2.8)$ \\
\hline Intron 22 inversion & $47(43.5)$ \\
\hline Large deletion & $5(4.6)$ \\
\hline Missense & $12(11.1)$ \\
\hline Nonsense & $11(10.2)$ \\
\hline Small deletion & $14(13.0)$ \\
\hline Small duplication & $6(5.6)$ \\
\hline Splice site mutation & $4(3.7)$ \\
\hline No mutation found & $2(1.9)$ \\
\hline Missing & $4(3.7)$ \\
\hline \multicolumn{2}{|l|}{ F8 mutation type, ${ }^{a} N(\%)$} \\
\hline Null mutations & $90(88.2)$ \\
\hline Non-null mutations ${ }^{\mathrm{b}}$ & $12(11.8)$ \\
\hline Family history of haemophilia, N (\%) & $42(38.9)$ \\
\hline Family history of inhibitors, $N(\%)$ & $13\left(31.0^{c}\right)$ \\
\hline
\end{tabular}

Abbreviation: IQR, interquartile range.

aData for 102 patients with genotype classification.

${ }^{\mathrm{b}}$ All non-null mutations were missense mutations.

${ }^{\mathrm{c}}$ Percentage of those with a family history of haemophilia.

effect on the incidence of inhibitors up to ED 35 or over the whole study period (data not shown).

\section{Discussion}

We present the final results on inhibitor development in the NuProtect study with simoctocog alfa, a fourth-generation human cell-line-derived rFVIII. This analysis reports inhibitor data for 105 PUPs treated with simoctocog alfa, making it the largest prospective study in true PUPs with a single FVIII product. The absolute incidence of all and high-titre inhibitors in NuProtect was 26.7 and 16.2\%, respectively. The cumulative incidence of all and high-titre inhibitors was 27.9 and $17.6 \%$, respectively. These rates are considerably

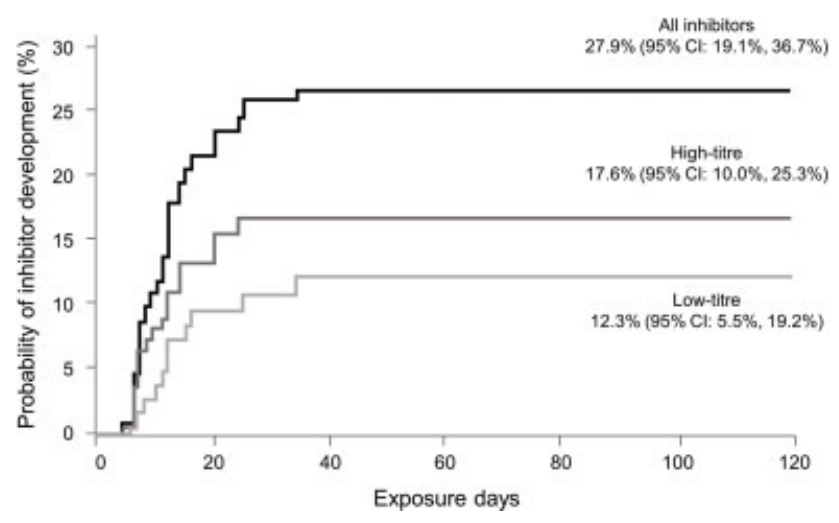

Fig. 2 Cumulative incidence of inhibitor development. Kaplan-Meier estimate.

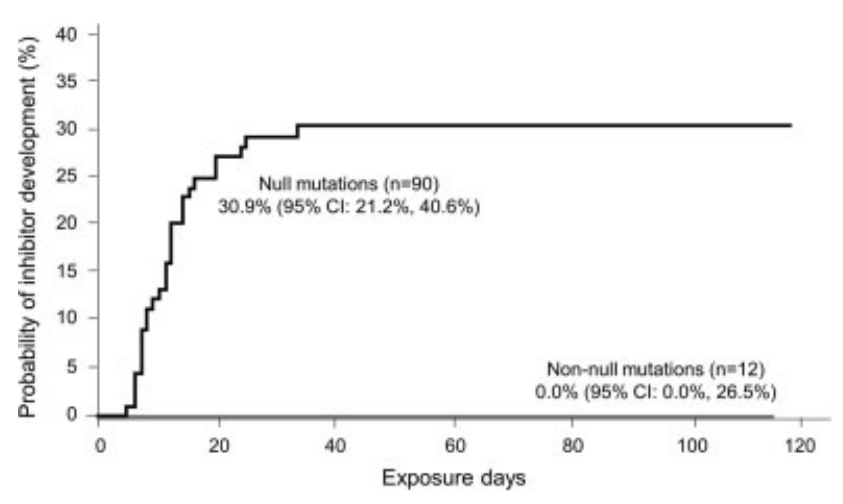

Fig. 3 Cumulative incidence of inhibitor development by null or nonnull F8 gene mutation. Kaplan-Meier estimate.

lower than the rates reported for the rFVIII arm in the SIPPET study, all derived from hamster cell lines (cumulative incidence $44.5 \%$ for all inhibitors and $28.4 \%$ for high-titre inhibitors), and in line with results obtained for the pdFVIII arm of the SIPPET study (26.8 and $18.6 \%$ for all and high-titre inhibitors, respectively). ${ }^{21}$

Recently published phase III PUP trials ${ }^{32,33}$ addressing the risk of inhibitor development with other single rFVIII products (all derived from hamster cell lines) have reported inhibitor rates in line with those reported for the rFVIII arm of the SIPPET study and hence higher than those reported with simoctocog alfa in our study. In a study of 58 PUPs treated with turoctocog alfa (NovoEight), 25 (43.1\%) developed inhibitors of which 16 (27.6\%) were high titre. ${ }^{32}$ In a study of 23 PUPs treated with single-chain rFVIII (Afstyla), 12 (52\%) developed inhibitors of which 6 (26\%) were high titre. ${ }^{33}$

F8 mutation type had an influence on the risk of inhibitor development in our study as has been previously reported. ${ }^{13}$ None of the 12 patients with non-null F8 mutations developed inhibitors with simoctocog alfa, which is consistent with what was reported for patients treated with pdFVIII in a post-hoc analysis of SIPPET data. ${ }^{34}$ Among SIPPET patients with null F8 mutations, the cumulative incidence of inhibitors was 31\% in 101 patients treated with pdFVIII and $47 \%$ in 96 patients treated with rFVIII. Among SIPPET patients with non-null F8 mutations, no inhibitors developed in 16 patients receiving pdFVIII treatment, whereas the 


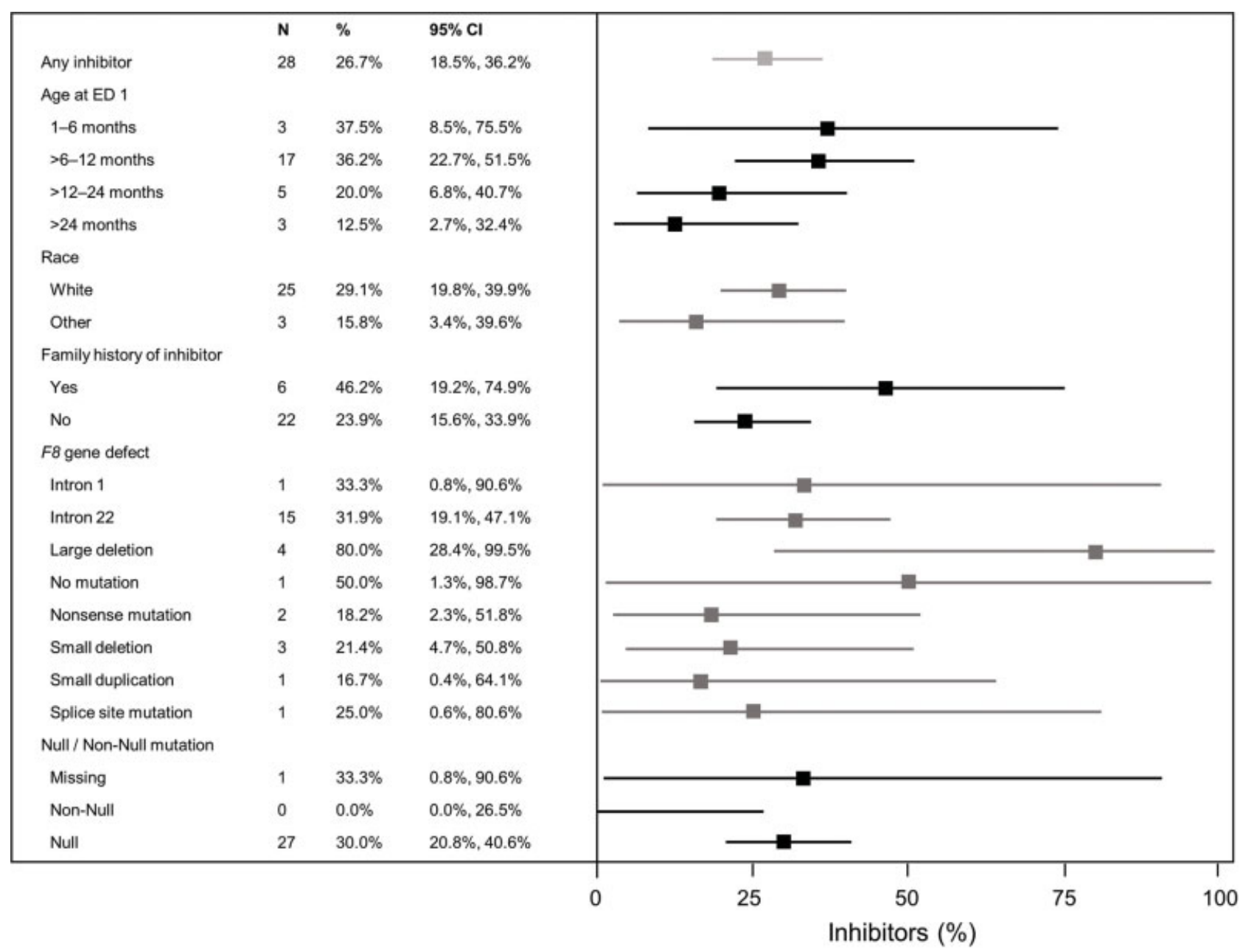

Fig. 4 Incidence of inhibitors in subgroups. Forest plot of absolute incidence percentages including exact $95 \%$ confidence interval.

cumulative incidence of inhibitors was $43 \%$ in 22 patients treated with rFVIII. ${ }^{34}$ These results suggest that simoctocog alfa appears to follow the pattern exhibited by pdFVIII concentrates rather than that of the hamster-cell derived rFVIII concentrates ( - Fig. 5). Notably, the distribution of F8 mutations was similar in NuProtect and SIPPET (-Fig. 6).

There is evidence in the literature that a family history of inhibitors is associated with an increased risk of inhibitor development. ${ }^{35}$ In NuProtect, the incidence of inhibitors in patients with a family history of inhibitors $(46.2 \% ; n=13)$ was double that of patients without a family history $(23.9 \%$; $n=92$ ). This difference was not statistically significant, but this may have been influenced by the relatively low number of patients with a family history of inhibitors.

In NuProtect, age $\leq 12$ months at first treatment was an independent prognostic factor for the development of inhibitors. PUPs aged 1 to 6 months at first treatment had the highest risk and those aged $>24$ months had the lowest risk for developing inhibitors. There are conflicting published data regarding the impact of age at first treatment on the risk for inhibitor formation. In two cohort studies, the risk of inhibitor development was higher in younger patients, but these studies were not controlled for other risk factors. ${ }^{36,37}$ Later studies that adjusted for confounders did not report an effect of age on inhibitor risk. ${ }^{16,38}$

There is also some evidence in the literature to suggest that treatment-related factors, particularly intensity of treatment, influence inhibitor development. ${ }^{14,39}$ In our study, treatment-related factors did not have a significant effect on inhibitor risk, which we speculate might be indicative of a lower immunogenic potential of Nuwiq.

A limitation of the NuProtect study is that almost all patients (95.4\%) enrolled across 17 countries were white or Asian, and no patients of African origin who have been reported to be at higher risk of inhibitor development ${ }^{40,41}$ were enrolled. A strength of the study is the inclusion of only true PUPs, i.e., without prior exposure to FVIII concentrates or any other blood products that may otherwise have mitigated or confounded the risk attributable to the study product. In addition, extensive immunogenicity testing was done throughout the study and all analyses were performed at a central laboratory.

In conclusion, the data from the NuProtect study show that PUPs with severe haemophilia A treated with 


\section{A. All inhibitors}

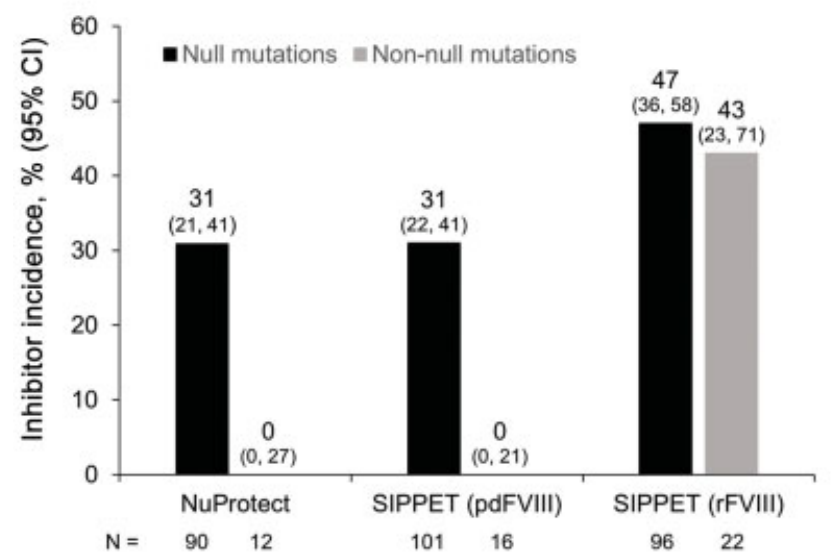

B. High-titre inhibitors

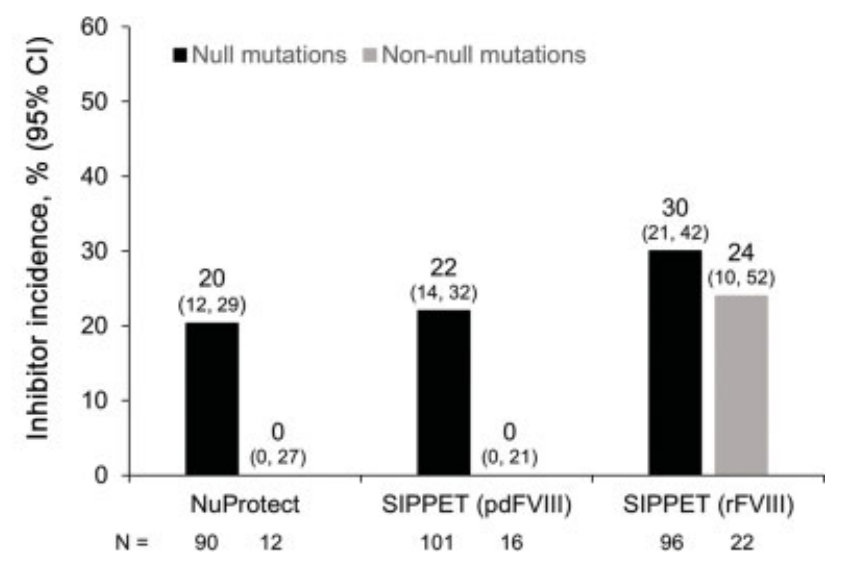

Fig. 5 Cumulative inhibitor incidence in the current study (NuProtect) and the SIPPET ${ }^{34}$ study. Data are shown by F8 mutation (null or non-null) for all inhibitors (A) and high-titre inhibitors (B).

A

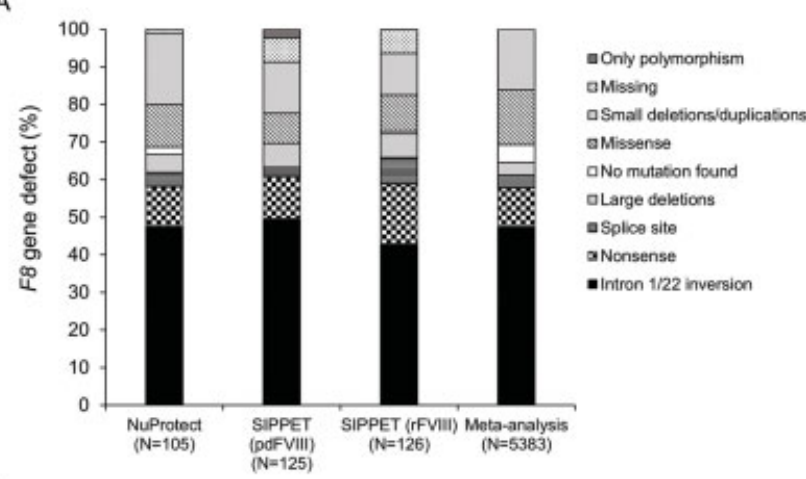

B

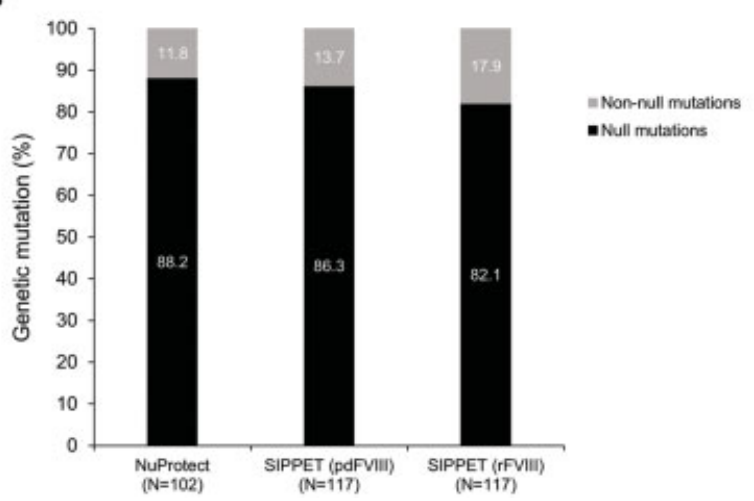

Fig. 6 Distribution of genetic defects. (A) F8 gene defect in the current study (NuProtect), the SIPPET study, ${ }^{21}$ and a meta-analysis ${ }^{13}$ of patients with severe haemophilia $A$ and (B) percentage null or nonnull mutations in NuProtect and SIPPET. ${ }^{21}$

simoctocog alfa had a lower high-titre inhibitor rate than that reported for hamster-cell-derived rFVIII products in other recent clinical trials and no inhibitors were observed in patients with non-null F8 gene mutations.

\section{What is known about this topic?}

- FVIII inhibitor development in previously untreated patients (PUPs) is the most serious contemporary treatment complication in haemophilia A.

- FVIII inhibitors develop in up to $40 \%$ of PUPs and have a detrimental impact on bleeding rates, quality of life and treatment costs.

\section{What does this paper add?}

- The NuProtect study was a multinational, prospective, open-label, phase III study investigating the immunogenicity of simoctocog alfa (Nuwiq), a recombinant FVIII produced in a human cell line, in PUPs.

- Inhibitors developed in $26.7 \%$ of 105 PUPs treated with simoctocog alfa; high-titre inhibitors in $16.2 \%$.

- The NuProtect study is the largest prospective study in PUPs with a single FVIII product.

- The study demonstrates that PUPs with severe haemophilia A treated with simoctocog alfa had a lower rate of high-titre inhibitor development than that reported for rFVIII products in other recent clinical trials.

\section{Author Contribbutions}

R. J. Liesner designed the research, analysed the data and wrote the first draft of the manuscript. All authors provided input, reviewed and approved the manuscript.

Funding

This trial was sponsored by Octapharma AG (Lachen, Switzerland) with medical writing assistance provided 
by nspm Itd, Meggen, Switzerland, and funded by Octapharma AG.

\section{Conflict of Interest}

R. J. Liesner, A. Abraham, C. Altisent, M. J. Belletrutti, M. Carcao, M. Carvalho, H. Chambost, A. K. C. Chan, L. Dubey, J. Ducore, M. Gattens, P. Gresele, Y. Gruel, B. Guillet, V.J. Yuste, L. Kitanovski, A. Klukowska, S. Lohade, J. Oldenburg, A. Pavlova, B. Pollio, M. Sigaud, V.Vdovin, K. Vilchevska, J. K. M. Wu and E. J. Neufeld were clinical study investigators for the NuProtect Study (Octapharma-sponsored). R. J. Liesner has received grants/research support from Octapharma, Bayer, Baxalta, Novo Nordisk and Roche, has acted as a consultant for Bayer and Baxalta, and has participated in speaker bureaus for Novo Nordisk, Octapharma and SOBI. A. Abraham has received grants/research support from Novo Nordisk and Roche, and has received support for attending scientific meetings from Novo Nordisk. C. Altisent has been a member of advisory boards and/or speaker bureaus for Baxalta (Shire), Bayer, CSL Behring, Grifols, Octapharma, Novo Nordisk, Pfizer, Roche and SOBI. M. J. Belletrutti has received research support from Octapharma, has acted as a consultant for Roche Canada, Sanofi and Takeda Canada, and has participated in speaker bureaus for Octapharma Canada and Takeda Canada. M. Carcao received research support/ grants from Bayer, Novo Nordisk, Pfizer, Sanofi and Takeda, and has participated in speaker bureaus for Bayer, CSL Behring, Grifols, LFB, Novo Nordisk, Octapharma, Pfizer, Roche, Sanofi and Takeda. M. Carvalho has been an investigator on clinical trials sponsored by CSL Behring, Novo Nordisk, Octapharma and Roche, and has received support for attending scientific meetings and honoraria (speaker fees/consultant in advisory boards) for Bayer, Baxalta (Shire), CSL Behring, Novo Nordisk, Octapharma, Pfizer, Siemens, SOBI and Stago. H. Chambost has received research support from CSL Behring, LFB, Novo Nordisk, Octapharma, Roche/ Chugaï, Shire/Takeda, SOBI; honoraria from Bayer, LFB, Octapharma, Pfizer, Roche and SOBI. A. K. C. Chan has been an investigator on clinical trials sponsored by Bayer, Biogen, CSL, Novo Nordisk, Octapharma and Shire and has received grants/research support from Bayer and Pfizer, and has participated in advisory boards for Bayer, Biogen, BioMarin, Novo Nordisk and Octapharma. J. Ducore has been an investigator on clinical trials sponsored by Octapharma, CSL Behring, OPKO Biologics, Bayer, Baxalta (Shire), Sparks Therapeutics, Biogen, Pfizer, Genentech (Roche), LFB and RevBio, has provided consultancy services to Octapharma, Bayer, Baxalta (Shire), Biogen and LFB, and is a speaker for Bayer. Y. Gruel has received support for attending scientific meetings and honoraria (speaker fees/consultant in advisory boards) from Baxalta (Shire), Bayer Healthcare, CSL Behring, LFB, Novo Nordisk, Octapharma, Pfizer, Roche and SOBI, has been an investigator in studies sponsored by Biogen, LFB and SOBI, and has received research support from CSL Behring, LFB and Octapharma. B. Guillet has been an investigator on clinical trials, or has received honoraria for speaking/consulting or funds for research from Bayer, CSL Behring, LFB, Novo Nordisk, Octapharma, Roche, SOBI and Takeda/Shire. V.
J. Yuste has received reimbursement for attending symposia/ congresses and/or honoraria for speaking and/or honoraria for consulting, and/or funds for research from Shire, Bayer, CSL Behring, Grifols, Novo Nordisk, SOBI, Octapharma and Pfizer. A. Klukowska has received personal fees from CSL Behring, Novo Nordisk, Octapharma, Pfizer, Roche, Shire and SOBI.M. E. Mancuso has received speaker and/or consultancy fees from Bayer Healthcare, CSL Behring, Takeda, Octapharma, Roche, Pfizer, Kedrion, Grifols, Novo Nordisk and SOBI.J. Oldenburg has received reimbursement for attending symposia/congresses or honoraria for speaking or honoraria for consulting, or funds for research from Bayer, Biogen Idec, Biotest, Chugai, CSL Behring, Grifols, Novo Nordisk, Octapharma, Pfizer, Roche, Shire and SOBI. A. Pavlova has participated in studies sponsored by Octapharma AG. B. Pollio has received support for attending scientific meetings and honoraria (speaker fees/consultant in advisory boards) from Baxalta (Shire), Bayer Healthcare, CSL Behring, Kedrion, Novo Nordisk, Octapharma, Pfizer and SOBI. M. Sigaud has received reimbursement for attending symposia/congresses, honoraria for speaking or for consulting from Biomarin, CSL Behring, Novo Nordisk, Octapharma, Roche and Shire/ Takeda. J. K. M. Wu received funding to attend meetings and has participated in Octapharma-funded clinical research. M. Jansen is a full-time employee of Octapharma $\mathrm{GmbH}$, Vienna, Austria. L. Belyanskaya, O. Walter and S. Knaub are employees of Octapharma AG, Lachen, Switzerland. E. J. Neufeld has received honoraria and participated in advisory boards for Octapharma. He has been a consultant for Genentech and Pfizer, and has participated in advisory boards for Novo Nordisk, Kedrion, Genentech, Baxalta/Shire (now Takeda), Novartis and CSL-Behring during the course of the NuProtect study. He has served on data monitoring committees for Bayer, Acceleron Pharma and ApoPharma (now Chiesi), and received research funding from the American Thrombosis and Hemostasis Network (ATHN). L. Dubey, M. Gattens, P. Gresele, L. Kitanovski, S. Lohade, V. Vdovin and K. Vilchevska declare no competing financial interests.

\section{Acknowledgments}

We thank patients/caregivers for their participation and all trial personnel and investigators (including Marina Abashidze, Irmel Alaya, Olga Aleinikova, Shashikant Apte, Annie Borel-Derlon, Mohamed El Khorassani, Jörg Faber, Annie Harroche, Silvia Horneff, Natalya Kavardakova, Christoph Königs, Thierry Lambert, Dinesh Nayak, Alexandra Russo, Amparo Santamaria and Valentin Turea).

\section{References}

1 Konkle BA, Huston H, Fletcher SN. Hemophilia A. In: Adam MP, Ardinger HH, Pagon RA, et al., eds. GeneReviews ${ }^{\circledR}$ [Internet]. Seattle, WA: University of Washington, Last updated 2017. Available at: https://www.ncbi.nlm.nih.gov/books/NBK1404/

2 Srivastava A, Santagostino E, Dougall A, et al. WFH guidelines for the management of hemophilia, 3rd edition. Haemophilia 2020; 26:1-158

3 Makris M. Prophylaxis in haemophilia should be life-long. Blood Transfus 2012;10(02):165-168 
4 Goudemand J, Peyvandi F, Lacroix-Desmazes S. Key insights to understand the immunogenicity of FVIII products. Thromb Haemost 2016;116(Suppl 1):S2-S9

5 van den Berg HM, Fischer K, Carcao M, et al;PedNet Study Group. Timing of inhibitor development in more than 1000 previously untreated patients with severe hemophilia A. Blood 2019;134 (03):317-320

6 Carcao M, Escuriola-Ettingshausen C, Santagostino E, et al;Future of Immunotolerance Treatment Group. The changing face of immune tolerance induction in haemophilia A with the advent of emicizumab. Haemophilia 2019;25(04):676-684

7 Morfini M, Haya S, Tagariello G, et al. European study on orthopaedic status of haemophilia patients with inhibitors. Haemophilia 2007;13(05):606-612

8 Mahlangu J, Oldenburg J, Callaghan MU, et al. Health-related quality of life and health status in persons with haemophilia $\mathrm{A}$ with inhibitors: a prospective, multicentre, non-interventional study (NIS). Haemophilia 2019;25(03):382-391

9 Di Minno MN, Di Minno G, Di Capua M, Cerbone AM, Coppola A. Cost of care of haemophilia with inhibitors. Haemophilia 2010;16 (01):e190-e201

10 Walsh CE, Jiménez-Yuste V, Auerswald G, Grancha S. The burden of inhibitors in haemophilia patients. Thromb Haemost 2016;116 (Suppl 1):S10-S17

11 Santagostino E, Young G, Escuriola Ettingshausen C, JimenezYuste V, Carcao M. Inhibitors: a need for eradication? Acta Haematol 2019;141(03):151-155

12 Oldenburg J, Lacroix-Desmazes S, Lillicrap D. Alloantibodies to therapeutic factor VIII in hemophilia A: the role of von Willebrand factor in regulating factor VIII immunogenicity. Haematologica 2015;100(02):149-156

13 Gouw SC, van den Berg HM, Oldenburg J, et al. F8 gene mutation type and inhibitor development in patients with severe hemophilia A: systematic review and meta-analysis. Blood 2012;119 (12):2922-2934

14 Peyvandi F, Garagiola I. Product type and other environmental risk factors for inhibitor development in severe hemophilia A. Res Pract Thromb Haemost 2018;2(02):220-227

15 Goudemand J, Laurian Y, Calvez T. Risk of inhibitors in haemophilia and the type of factor replacement. Curr Opin Hematol 2006;13(05):316-322

16 Chalmers EA, Brown SA, Keeling D, et al;Paediatric Working Party of UKHCDO. Early factor VIII exposure and subsequent inhibitor development in children with severe haemophilia A. Haemophilia 2007;13(02):149-155

17 Mancuso ME, Mannucci PM, Rocino A, Garagiola I, Tagliaferri A, Santagostino E. Source and purity of factor VIII products as risk factors for inhibitor development in patients with hemophilia A.J Thromb Haemost 2012;10(05):781-790

18 Gouw SC, van der Bom JG, Auerswald G, Ettinghausen CE, Tedgård $\mathrm{U}$, van den Berg HM. Recombinant versus plasma-derived factor VIII products and the development of inhibitors in previously untreated patients with severe hemophilia A: the CANAL cohort study. Blood 2007;109(11):4693-4697

19 Gouw SC, van der Bom JG, Ljung R, et al;PedNet and RODIN Study Group. Factor VIII products and inhibitor development in severe hemophilia A. N Engl J Med 2013;368(03):231-239

20 Fischer K, Lassila R, Peyvandi F, et al;EUHASS participants. Inhibitor development in haemophilia according to concentrate. Fouryear results from the European HAemophilia Safety Surveillance (EUHASS) project. Thromb Haemost 2015;113(05):968-975

21 Peyvandi F, Mannucci PM, Garagiola I, et al. A randomized trial of factor VIII and neutralizing antibodies in hemophilia A. N Engl J Med 2016;374(21):2054-2064

22 Lieuw K. Many factor VIII products available in the treatment of hemophilia A: an embarrassment of riches? J Blood Med 2017;8:67-73
23 Casademunt E, Martinelle K, Jernberg M, et al. The first recombinant human coagulation factor VIII of human origin: human cell line and manufacturing characteristics. Eur J Haematol 2012;89 (02):165-176

24 Kannicht C, Ramström M, Kohla G, et al. Characterisation of the post-translational modifications of a novel, human cell linederived recombinant human factor VIII. Thromb Res 2013;131 (01):78-88

25 Sandberg H, Kannicht C, Stenlund P, et al. Functional characteristics of the novel, human-derived recombinant FVIII protein product, human-cl rhFVIII. Thromb Res 2012;130(05):808-817

26 Winge S, Yderland L, Kannicht C, et al. Development, upscaling and validation of the purification process for human-cl rhFVIII $\left(\right.$ Nuwiq $^{\circledR}$ ), a new generation recombinant factor VIII produced in a human cell-line. Protein Expr Purif 2015;115:165-175

27 Brooks SA. Appropriate glycosylation of recombinant proteins for human use: implications of choice of expression system. Mol Biotechnol 2004;28(03):241-255

28 Walsh G, Jefferis R. Post-translational modifications in the context of therapeutic proteins. Nat Biotechnol 2006;24(10):1241-1252

29 Lissitchkov T, Klukowska A, Pasi J, et al. Efficacy and safety of simoctocog alfa $\left(\right.$ Nuwiq $\left.^{\circledR}\right)$ in patients with severe hemophilia A: a review of clinical trial data from the GENA program. Ther Adv Hematol 2019;10:2040620719858471

30 Liesner RJ, Abashidze M, Aleinikova O, et al. Immunogenicity, efficacy and safety of Nuwiq ${ }^{\circledR}$ (human-cl rhFVIII) in previously untreated patients with severe haemophilia A - Interim results from the NuProtect Study. Haemophilia 2018;24(02):211-220

31 Verbruggen B, Novakova I, Wessels H, Boezeman J, van den Berg M, Mauser-Bunschoten E. The Nijmegen modification of the Bethesda assay for factor VIII:C inhibitors: improved specificity and reliability. Thromb Haemost 1995;73(02):247-251

32 Yaish H, Matsushita T, Belhani M, et al. Safety and efficacy of turoctocog alfa in the prevention and treatment of bleeds in previously untreated paediatric patients with severe haemophilia A: Results from the guardian 4 multinational clinical trial. Haemophilia 2020;26(01):64-72

33 Santagostino E, Koenigs C, Djambas Khayat C, et al. Interim analysis of the extension study with rVIII-SingleChain in previously untreated patients (PUPs) with severe hemophilia A (CSL627-3001). Blood 2019;134(Suppl 1):162

34 Rosendaal FR, Palla R, Garagiola I, Mannucci PM, Peyvandi FSIPPET Study Group. Genetic risk stratification to reduce inhibitor development in the early treatment of hemophilia A: a SIPPET analysis. Blood 2017;130(15):1757-1759

35 Bachelet D, Albert T, Mbogning C, et al;ABIRISK consortium. Risk stratification integrating genetic data for factor VIII inhibitor development in patients with severe hemophilia A. PLoS One 2019;14(06): e0218258

36 Lorenzo JI, López A, Altisent C, Aznar JA. Incidence of factor VIII inhibitors in severe haemophilia: the importance of patient age. Br J Haematol 2001;113(03):600-603

37 van der Bom JG, Mauser-Bunschoten EP, Fischer K, van den Berg HM. Age at first treatment and immune tolerance to factor VIII in severe hemophilia. Thromb Haemost 2003;89(03):475-479

38 Santagostino E, Mancuso ME, Rocino A, et al. Environmental risk factors for inhibitor development in children with haemophilia A: a case-control study. Br J Haematol 2005;130(03):422-427

39 Garagiola I, Palla R, Peyvandi F. Risk factors for inhibitor development in severe hemophilia A. Thromb Res 2018;168:20-27

40 Miller $\mathrm{CH}$, Benson J, Ellingsen D, et al;Hemophilia Inhibitor Research Study Investigators. F8 and F9 mutations in US haemophilia patients: correlation with history of inhibitor and race/ ethnicity. Haemophilia 2012;18(03):375-382

41 Miller CH. Game, set, match for factor VIII mismatch? Blood 2015; 126(07):829-830 